HEART FAILURE

\section{ACTRII signalling implicated in HF}

The activin receptor type 2 (ACTRII) pathway is activated in ageing and heart failure (HF), and inhibition of this catabolic pathway is a potential therapeutic target for HF. "What's particularly exciting to us is that "human versions' of the two inhibitors we used for these studies have already been used in early-phase clinical trials for other indications, so we're hopeful that they - or other inhibitors could be re-purposed for HF," comments Anthony Rosenzweig, senior investigator on the article now published in Sci. Transl Med.

First author, Jason Roh, and colleagues showed that circulating levels of follistatinrelated protein 3 (FSTL3), a downstream regulator of ACTRII signalling and an indirect measure of pathway activity, are increased with human ageing, frailty and HF severity. In mice, increasing the circulating levels of activin A, a ligand for ACTRII, increased cardiac ACTRIl signalling and impaired cardiac function. Of note, a murinized version of bimagrumab (CDD866), a monoclonal antibody that blocks ACTRIIA and ACTRIIB and which is currently under clinical testing for sarcopenia, improved subclinical left ventricular systolic dysfunction in aged mice. Moreover, in a mouse model of $\mathrm{HF}$ induced by transverse aortic constriction (TAC), CDD866 improved systolic function and reduced pulmonary oedema. Cardiomyocyte-specific Acvr $2 b^{-/-}$(encoding ACTRIIB) mice were protected from systolic dysfunction after TAC. Finally, the investigators showed that one mechanism of action of ACTRII signalling might be via $\mathrm{E} 3$ ubiquitin-protein ligase SMURF1-dependent ubiquitination and subsequent proteasome-dependent degradation of SERCA2, a vital component of the cardiomyocyte $\mathrm{Ca}^{2+}$-handling machinery.

"Activin signalling ... could help the energy-starved failing heart to compensate early on by inhibiting growth and ATP utilization, but chronic activation becomes deleterious," suggests Rosenzweig, similar to the maladaptive activation of the reninangiotensin-aldosterone system in HF. "Disrupting this downward spiral mediates substantial benefit in animal models, and we hope to test whether similar benefits could be attained in clinical HF," he concludes.

\title{
Novel vasodilatory factor identified
}

Singlet molecular oxygen $\left({ }^{1} \mathrm{O}_{2}\right)$, a reactive oxygen species with well-known functions in plants, bacteria and fungi, also has an important pathophysiological role in mammals. According to a new study, ${ }^{1} \mathrm{O}_{2}$ generated by endothelial indoleamine 2,3-dioxygenase 1 (IDO1) is involved in the redox regulation of arterial relaxation and blood pressure during inflammatory conditions through formation of a tryptophan (Trp)-derived metabolite.

Previous work from the same group had identified another product of IDO1-mediated Trp metabolism, kynurenine (Kyn), as a factor contributing to blood-vessel relaxation in inflammatory conditions. However, Stanley et al. found that recently purchased or highly purified Kyn did not induce vasodilatation. By contrast, purified Trp in the presence of IDO1 caused blood-vessel relaxation, suggesting that another vasodilator was being formed and was present as a contaminant in early commercial preparations of Kyn. The research team set out to find the vasodilator and identified a compound, cis-WOOH, that was generated by IDO1 in a reaction involving $\operatorname{Trp}$ and ${ }^{1} \mathrm{O}_{2}$ in the presence of hydrogen peroxide.
Both IDO1 and ${ }^{1} \mathrm{O}_{2}$ were required for cis-WOOH formation and arterial relaxation. Under inflammatory conditions in vivo, endothelial IDO1 formed ${ }^{1} \mathrm{O}_{2}$ followed by the generation of $c i s-\mathrm{WOOH}$, which acted as a signalling molecule inducing arterial relaxation and decreasing blood pressure. These effects were dependent on oxidative activation of protein kinase G1 $\alpha$ (PKG1 $\alpha$ ). cis-WOOH activated PKG1 $\alpha$ by oxidizing a specific cysteine residue (Cys 42), which leads to PKGla dimerization.

Finally, Stanley et al. demonstrated that IDO1 was also expressed in arteries with atherosclerosis and in resistance arteries in $A$ poe $^{-/-}$mice and contributed to the regulation of blood pressure, suggesting that the IDO1-PKG1 $\alpha$ axis has a role in blood-pressure control in atherosclerosis. By contrast, this pathway had no role in cardiac tissue after cardiac pressure overload in mice.

Irene Fernández-Ruiz

ORIGINAL ARTICLE Stanley, C. P. et al. Singlet molecular oxygen regulates vascular tone and blood pressure in inflammation. Nature https://doi.org/10.1038/s41586-0190947-3 (2019)

\section{CARDIOMYOPATHIES}

\section{Brain-heart axis in Takotsubo syndrome}

Functional connectivity in the limbic system of the brain, which controls emotion and the autonomic system, is reduced in patients with Takotsubo syndrome compared with healthy individuals. This finding comes from a study using resting-state functional MRI, now published in European Heart Journal.

Investigators recruited 15 women with Takotsubo syndrome (only $~ 10 \%$ of cases of Takotsubo syndrome occur in men) and 39 age-matched, female control individuals and assessed resting state functional connectivity in the brain - that is, the extent to which two brain regions are simultaneously activated, which indicates that they are communicating. Four different sets of brain network nodes were assessed: sympathetic, parasympathetic, default mode and whole brain.

Resting-state functional connectivity was reduced in the sympathetic, parasympathetic and default networks in patients with Takotsubo syndrome compared with healthy control individuals. In addition, the assessment of the whole-brain network identified hypoconnectivity in the limbic system, which includes areas such as the amygdala, cingulate gyrus, hippocampus and insula that are involved in emotional processing and control of the autonomic nervous system and cardiac function. In addition, the cingulate gyrus is an important structure in depression, which is common in patients with Takotsubo syndrome. Of note, the investigators had previously identified structural alterations in patients with Takotsubo syndrome in the same regions of the brain in which functional hypoconnectivity was identified in this study.

"While there are likely [to be] many causes of Takotsubo syndrome ... the present findings suggest that alterations in the function of certain parts of the central nervous system may result in the onset of a Takotsubo syndrome event in response to a stressful trigger," conclude the researchers.

\section{Gregory B. Lim}

ORIGINAL ARTICLE Templin, C. et al. Altered limbic and autonomic processing supports brain-heart axis in Takotsubo syndrome. Eur. Heart J. https://doi.org/10.1093/eurheartj/ ehz068 (2019)

FURTHER READING Kastaun, S. et al. Psychosocial and psychoneuroendocrinal aspects of Takotsubo syndrome. Nat. Rev. Cardiol. 13, 688-694 (2016) 